\title{
A Study of the Effects of Playful Positive Aging Groups on the Well-Being of Taiwan's Elderly Incorporating Six Bricks and Play Box
}

\author{
Pay-Ling Harn, Yu-De Zhang, Ping Jen, Jia-Syun LEE, Hsin-Wen Chen
}

\begin{abstract}
This study aims to integrate Six Bricks and Play Box to develop a playful positive aging groupand further explore the effects of research intervention on the well-being of the elderly. The study involved 10 elderly Taiwanese, including 7 women and 3 male elders. The research participants accepted a playfulpositive aging group with a six-week, two-hour weekly and implemented "well-being scale for elders" before and after the group began and ended. The results showed that the mean of the researchparticipants in the pre-test of the "well-being scale for elders" was 3.95, with a standard deviation of 0.33 . The post-test mean is 4.31 , the standard deviation is $\mathbf{0 . 4 5}$, the difference between the pre- and post-test averages is 0.39 , the $t$-value is $\mathbf{- 2 . 7 5}$, the $p$-value is 0.02 , and the $p<0.05$, which is a significant difference. Therefore, the playfulpositive aging group showedan immediate effect on the well-being of the elderly. Based on the results of the study, the paper puts forward some suggestions for the research and practice of positive aging in the future.
\end{abstract}

Index Terms-Playful approach, positive aging, Six Bricks, Play Box, well-being

\section{INTRODUCTION}

At present, Taiwan is facing the problem of population aging. From 1993 to 2008, the proportion of Taiwan's elderly aged 65 and over in the total population also rose from 7.10 $\%$ to $14.05 \%$. Within 25 years, Taiwan had moved from an "ageing society" to an "age society"[1]. Living a long life is important, but how to live optimistically, with quality, happiness is worthier of attention. After entering old age, with the change of physical, psychological and social status, the elderly may have adjustment difficulties. As a result, old people may suffer from depression in their old age [2]. Therefore, in today's aged society, how to promote positive aging is an important issue.

Rowe and Kahn [3][4] state that positive aging must include three elements: avoiding disease and disability,

Pay-Ling Harn, Assistant Professor, Applied Psychology Department, Hsuan Chuang University, No.48, Hsuan Chuang Rd., Hsiang San District, Hsinchu City, Taiwan maintaining mental function, and engaging in society.Takentogether, it can be learned that positive aging is not only to slow down the rapid physiological degradation, but also to expand the positive aspects of social participation of the elderly, in order to promote the elderly's sense of self-meaning and value. Therefore, positive aging should cover three levels of physical health, psychological adaptation and social support.

According to the World Health Organization [5], positiveaging is defined as a complete state of physical, psychological and social well-being. The WHO's assessment of positive aging as an "objective state" may not reflect the "subjective feelings" of the elderly. The fact that an elderly person has no physical or mental illness and continues to participate in social activities does not necessarily mean that he is satisfied with his old age, nor does it mean that he can find self-worth and meaning in his old age life.This study holds that positive aging should be more of a self-evaluation close to life happiness and subjective well-being, rather than an optimal intersection of objective factors.

The five-element model of "social well-being" proposed by Keyes and Annas [6] is social integration, social acceptance, social contribution, social realization, and social coherence. Keyes and others argue that emotional well-being, psychological well-being, and social well-being should be integrated as an indicator of personal mental health. This study attempts to construct the constituent indicators of "positive aging" through the self-evaluation of subjective well-being (SWB) by the elderly.

In response to the needs of different levels of positive aging, elderly care workers have begun to develop different modes of promoting positiveaging. On the physiological level, communities or institutions will offer courses on body rhythm and memory activation to help the elderly maintain appropriate physiological functions. At the psychological level, there are reminiscence therapy, mindfulness, and painting therapy to promote the integration of the elderly and deepen their sense of meaning. At the social level, it has begun to promote intergenerational learning to deepen the connection between the elders and grandchildren, and the sense of family belonging. In addition, scholars elaborated on the use of positive psychological intervention to develop cognitive, volitional and behavioral activities and design intergenerational learning models [7]. 
In recent years, in the positive aging program, a new integrated positive psychological intervention has begun to be incorporated: the "Six Bricks and Play Box( SBPB ) ". Six Bricks and Play Box were developed in South Africa. They were originally used for children's learning. Now they have been extended to applications of different ages, ethnic groups and topics. Through A holistic approach to cognitive, creative, social, emotional and physical skills withSBPB, it is possible to fully design an integrated elderly program suitable for the elderly with physical, psychological and social support. The characteristics of bricks, such as tactile, auditory sense, and visual sense, have a promoting effect on brain development and memory enhancement [8]. Harn and Hsiao [9] pointed out that the Six Bricks and Play Box aging program are executable, integrated, and multi-effective, which can promote the physical health, psychological comfort and social support of the elderly.

In the playful positive aging program, through social group is the main form. Using social group, let the elderly obtain an optimistic and positive attitude from the group, provide psychological support and treatment, education and entertainment services for the elderly, solve the problems of the elderly's life adaptation, and prevent the occurrence of elderly problems [10]. This research adopted Six Bricks and Play Box (SBPB) playful approach, incorporates other programs that can promote positive relationships, positive emotions and positive vitality, to develop a six-week playful positive aging group, and explore the effect of the research program on the well-beingof the elderly.

Based on discussions above, the objectives of this study were to elucidate(a) developing the application mode of playfulpositive aging groups; and(b) the effect of playful aging group on the elders' well-being.

\section{METHOD}

\section{A. Playful Positive Aging Groupincorporating Six Bricks and Play Box}

The playful positive aging group is based on the three-dimensional concept of well-being (psychological well-being, emotional well-being, and social well-being) advocated by Keyes. It is a six-week elderly group that lasts two hours at a time. In this study, Six Bricks and Play Box's playful approach were used, and the activities of delicious food taste to enhance Sense of smellwas incorporated to promote positive emotions, positive relationships, and positive vitality programs to promote positive aging of the elderly. Establish positive relationships with others through a group approach, and have a life goal in the group to perceive that life is meaningful now and in the past. At the beginning of each group, exercise is used to promote physical health, such as the release of endorphins and other neurotransmitters, and the subsequent relaxation. The playful positive aginggroup of this study is shown in Table 1 .

The physical skills and memory training learningactivities of Six Bricks arehelpful to promote the muscle endurance and balance of the elderly. Balance ability is a positive benefit. The Tricky Tower is a typical physical skill learning activity of Six Bricks. As the height increases, it will produce a higher degree of pleasure and flow experience.
This activity requires concentration, communication, cooperation and problem-solving skills. This will use different senses and fine and gross movements to improve brain activity and muscle abilities. Neuroscience scholars point out that even if physical activity will have an immediate and positive impact on the brain, even if it cannot completely avoid the occurrence of Alzheimer's, Parkinson's disease, and depression, it can reduce the occurrence of these diseases. Negative influence. In our practical experience, by continuing to practice activities such as stopping dancing and the Tower of Strangeness, the elderly can maintain balance for an increase in time, which shows that their physical skills have improved, and its additional effect can increase the elderly's sense of self-efficacy [8].

In the experience of playful learning, it has a highly stimulating effect of pleasure, which may come from the colorful visual effects of Six Bricks and Play Box, the fun of activities, or the joy of successfully challenging tasks. All of these can promote the positiveness of the elderly. To effect. Using the play box to narrate the past, the future, and the present, or construct beautiful memories, allows the elderly to connect with others through narration, and to get close to and express their thoughts and emotions. This will have a more dynamic and visual effect than traditional life story narration [8][9].

Table 1.Playful Positive Aging Group

\begin{tabular}{|c|c|}
\hline Topic & Playful activities \\
\hline Positive connection & Yoga, social activities. \\
\hline Bridge of hope & $\begin{array}{l}\text { Tricky tower with Six Bricks, } \\
\text { moving castle with Play Box }\end{array}$ \\
\hline Playful stacking & Supporting task with \\
\hline & SixBricks, happiness log with \\
\hline Delicious food map & $\begin{array}{l}\text { Delicious food memories and } \\
\text { journeys }\end{array}$ \\
\hline Savor life & Life story with Play Box \\
\hline Playful achievement & STEAM Maker \\
\hline
\end{tabular}

\section{B. Participants}

The participants of this study were 10 elderly people over 50 years old in Taiwan, including 6 female and 4 male elders.

\section{Research Instruments}

(a) Well-being scale for the elderly. This study uses the Well-being scale for the elderly compiled by Lien and Cheng [11] to measure the participants' happiness. This scale was compiled with 332 elderly people as the research subjects. The full scale includes emotional well-being, psychological well-being and social well-being, with a total of 9 items. The reliability test of the scale is.91, which has acceptable reliability. The factor structure test is conducted by factor analysis, which has a good construct validity.

(b) Six Bricks and Play Box.The Six Bricks and Play Box are developed by a group of South African teachers. It's a playful 
learning concept that takes a holistic approach, emphasizing the importance of physical, cognitive, creative, social, emotional skills, and how these skills interact Influence and support. Six Bricks use the six-color DUPLO ${ }^{\circledR}$ bricks of red, yellow, orange, green, dark blue, and light blue to learn through play, perform quick daily exercises and activities, and help children develop in various fields. The Play Box is also a concept of playful learning. It uses mixed DUPLO ${ }^{\circledR}$ elements, including basic bricks, figures, animals, vehicles, plates and other objects that attract children to learn happily. It creates an easy for children to explore, practice and experience. An environment of interaction with others allows children to learn through play to promote children's holistic development.

\section{Date Analysis}

Before and after the group, the research participants performed the pre-test and post-test of the "Well-being scale for the elderly ". Researchers used t-tests of dependent samples to compare differences to explore the effect of playful positive aging group on the well-being of research participants.

\section{RESULT}

Before the start of the group in the first week and after the group in the sixth week, the study participants will fill out the " Well-being scale for the elderly " for pre-test and post-test. The researchers further compared the differences of the mean with the t-test of dependent samples to explore the influence of playful positive aging groups on the well-being of research participants. The mean in the pre-test was 3.95, the standard deviation was 0.33 ; the average in the post-test was 4.31 , the standard deviation was 0.45 , the difference between the pre- and post-test averages was 0.39 , the $t$ value was -2.75 , and $p<0.05$ (Table 2). It can be seen thatthe score of well-being achieved significant immediate effect.

difference in the pretest on each dimension of the "positive emotion scale for elementary students" and "elementary school student emotional competency scale". However, Table 3 shows that the experimental and control groups showed significant difference in the protest on each dimension of the "positive emotion scale for elementary students" and "elementary school student emotional competency scale". This result reveals that the playful positive psychology intervention significantly enhances the children's positive emotions and emotional competency.

Table2.t-test analysis table for dependent samples ( $\mathrm{N}=9$ )

\begin{tabular}{lcccccc}
\hline Variable & \multicolumn{2}{c}{ Pre-test } & \multicolumn{2}{c}{ Post-test } & $t$ & $p$ \\
& $\mathrm{M}$ & $\mathrm{SD}$ & $\mathrm{M}$ & $\mathrm{SD}$ & $d f=9$ & \\
\hline Well-being & 3.95 & 0.33 & 4.31 & 0.45 & $-2.75^{*}$ & .022 \\
\hline Note: $* p<.05$ & & & & & &
\end{tabular}

\section{COUNCLUSION}

This research is to explore the application mode of Six Bricks and Play Box, which was originally used for children's education, in positive aging. The Six Bricks and Play Box are a holistic playful learning, which promotes brain activation through touch and move, and promotes the positive development of physiology, psychology and social relationship. The design of this group is to promote the activation of brain learning, positive emotions, positive relationship and physical health through SBPB, which echoes the positive promotion of physical, psychological and social support for positive aging. According to the research results, the playful positive aging group can effectively improve the emotional, psychological and social well-being of the elderly. However, limited by research tools, it fails to evaluate the maintenance effect of the elderly's physical health and fails to cover the complete positive aging aspect. This will limit the inference of the positive aging effect of the playful positive aging group.

This study develops a playful positive aging group based on the holistic approach of Six Bricks and Play Box, and provides an innovative positive aging promotion model. This study develops a playful positive aging group based on the holistic approach of Six Bricks and Play Box, and provides an innovative positive aging promotion model. Six Bricks and Play Box 's positive aging program emphasizes the provision of physical, psychological and social all-round promotion. With the physiological characteristics of vision, tactileand auditory sense, as well as ease of use, creativity, simplicity and other use characteristics, Six Bricks and DUPLO® Play Box are highly developed in the positive aging and senior service[8][9].In the future, the concept of the playful positive aging group in this research can be used as a prototype to develop programs for the elderly with different characteristics. In this study, only the measurement of psychological and social well-being was included. In the follow-up research, the assessment of physical health can be increased, and the overall effect of positive aging can be more fully presented.

\section{REFERENCES}

[1] Ministry of the Interior (2018). Summary analysis of the survey on the situation of the elderly. Retrieved November 6, 2019, https://www.moi.gov.tw/stat/news_detail.aspx?sn=13742

[2] Hooyman, N. \&Kiyak, H. A.(1996). Social Gerontology: A multidisciplinary perspective. USA: Allyn \& Bacon.

[3] Rowe, J. W., \& Kahn, R. L. (1997). Successful aging. The Gerontologist, 37(4),433-440.

[4] Rowe, J. W., \& Kahn, R. L. (1998). The structure of successful aging. In J. W. Rowe\& R. L. Kahn (Eds.), Successful Aging (pp. 36-52). NewYork: Random House.

[5] WHO. (2002). Active aging a policy framework. Geneva: World Health Organization.

[6] Keyes, C. L. M., \& Annas, J. (2009). Feeling good and functioning well: Distinctive concepts in ancient philosophy and contemporary science. The Journal of Positive Psychology, 4, 197-201.

[7] Li, X. ( 2018 )。The effects of positive psychology intergenerational learning program on children's attitudes toward older people and older people's successful aging.Journal of STU , 20 ( 2 ) , 133-153。

[8] Harn, P.L., \& Hsiao, C.C.(2020).Playful well-being: Six Bricks and Play Box's positive psychological helping mode. Taipei: Hungyen.

[9] Harn, P.L., \& Hsiao, C.C.(2019). Healthy aging promotion mode of Six Bricks.Counseling \& Guidance, 408,36-39.

[10] Li, P. L. (2009).Action Research on the Reminiscence Group Work for Old People with Dementia(Unpublished master dissertation). Meiho University, Pin Tung, Taiwan.

[11] Lien, T.C., \& Cheng, H.F. (2015). The development of the well-being scale for elders in Taiwan.Guidance Quarterly,51(1), p29-37. 
Pay-Ling Harn received Ph.D. degree in educational psychology and counseling from Taiwan Normal University, Taipei, Taiwan. Currently, she is an assistant professor at Hsuan Chuang University, Hsinchu, Taiwan. She is certified counseling psychologist, Six Bricks and Play Box master trainer. Her research interests are in positive psychology, career counseling, and playful positive psychology interventions.

Yu-DeZhang, PingJen, Jia-SyunLEE and Hsin-Wen received Bachelor degree in applied psychology department from Hsuan Chuang University. 